\title{
Structural Anisotropy in a Crustacean Claw Calcified with Amorphous Calcium Phosphate
}

\author{
Miloš Vittori ${ }^{1}$, Vesna Srot $^{2}$, Birgit Bussmann², Peter A. van Aken² and Jasna Štrus ${ }^{1}$ \\ 1. Department of Biology, Biotechnical faculty, University of Ljubljana, Večna pot 111, 1000 Ljubljana, \\ Slovenia \\ 2. Stuttgart Center for Electron Microscopy, Max Planck Institute for Solid State Research, \\ Heisenbergstr. 1, 70569 Stuttgart, Germany
}

Animals form a variety of mineralized biological matrices that perform different functions, ranging from ion storage to protecting animal bodies and enabling movement. From the mechanical point of view, extracellular matrices forming animal skeletons are of particular interest, as they are evolutionary optimized for the function they perform. Understanding structure-function relationships of these natural composite materials can lead to the development of biomimetic designs with industrial applications.

The cuticle forming the crustacean exoskeleton is an example of an extracellular matrix with diverse mechanical functions. It is generally organized as a stack of sheets consisting of parallel chitin-protein fibers. In each sequential sheet, the fibers shift their orientation, resulting in a quasi-isotropic material [1]. The fibers are embedded in a matrix most often calcified with calcite and amorphous calcium carbonate [2].

The architecture and mineral composition of the cuticle forming different parts of a crustacean's body (Fig. 1A) are evolutionary optimized for the function they perform. An example are the claws of walking legs (Fig. 1B), which are thin structures supporting the entire body and are mostly subjected to unidirectional loads. Furthermore, the claws come into contact with the substrate, which subjects them to wear. We analyzed claws of the walking legs in the terrestrial crustacean Porcellio scaber with fieldemission scanning electron microscopy on a JEOL $7500 \mathrm{~F}$ microscope in order to elucidate the nanostructure of these high-performing skeletal elements. To further analyze their composition, energydispersive X-ray spectroscopy (EDX) and electron energy-loss spectroscopy (EELS) combined with scanning transmission electron microscopy (STEM) imaging at high spatial and high energy resolution were used to characterize these amazing architectures using Zeiss SESAM and JEOL ARM200F microscopes at different accelerating voltages.

Unlike other regions of the exoskeleton, which is mineralized with calcium carbonate, the predominant mineral component of the claw cuticle is amorphous calcium phosphate (Fig. 2). Our analyses showed that the claw endocuticle is highly structurally anisotropic, consisting of axially oriented chitin-protein fibers and amorphous calcium phosphate particles (Fig. 2B), whereas the exocuticle is non-calcified and highly brominated. These structural and compositional features of the claw cuticle likely confer the claw greater resistance to fracture and wear when exposed to axial loading. [3] 


\section{References:}

[1] Y Bouligand, Tissue \& Cell 4 (1972), pp. 189-217.

[2] R Roer and R Dillaman, American Zoologist 24 (1984), pp 893-909.

[3] The research leading to these results has received funding from the European Union Seventh

Framework Program [FP/2007-2013] under grant agreement No.312483 (ESTEEM2).
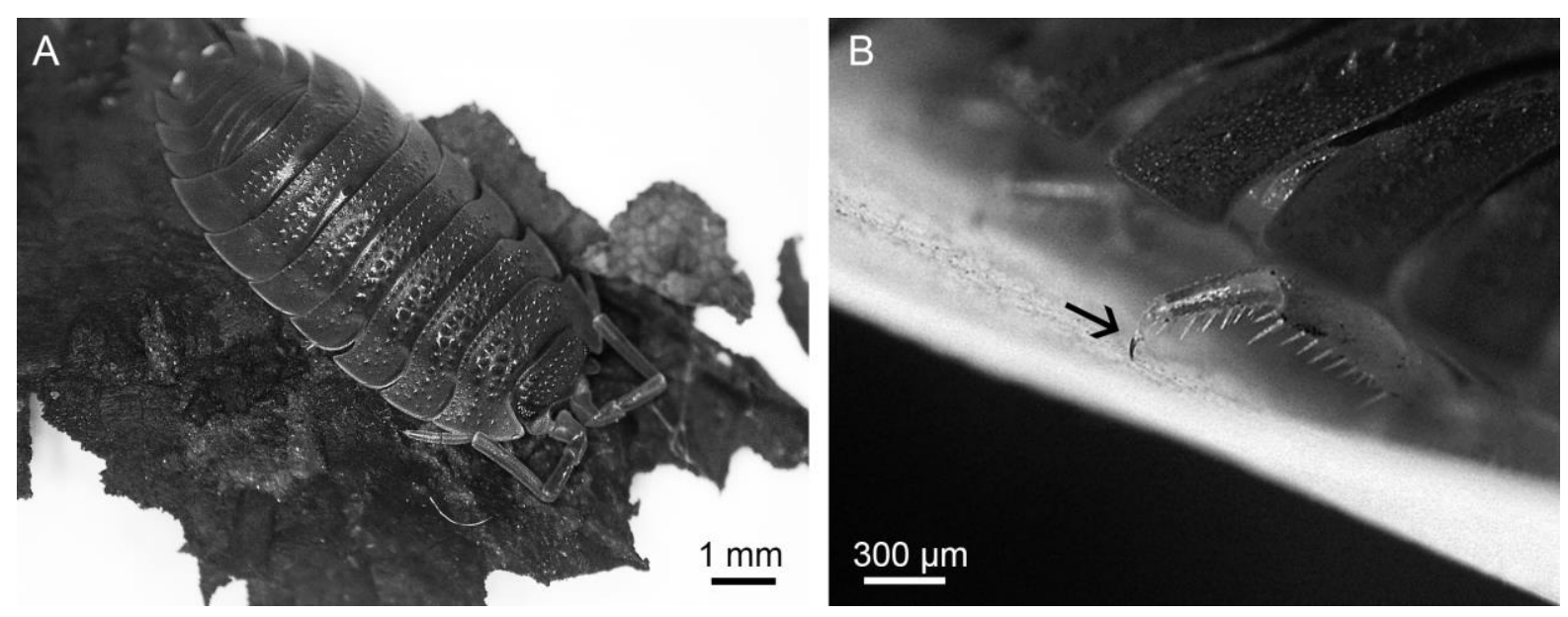

Figure 1. A: The terrestrial crustacean Porcellio scaber. B: One of the walking legs ending in a claw (arrow).

A

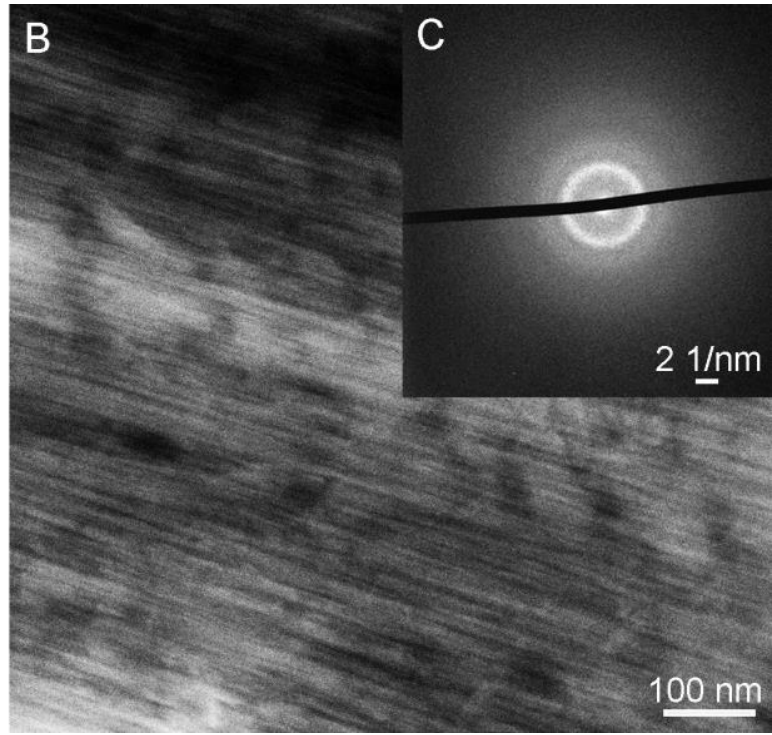

Figure 2. A: EDX spectrum obtained from the claw endocuticle indicating the presence of calcium phosphate. B: High-angle annular dark-field (HAADF)-STEM image of the claw endocuticle showing the unidirectional orientation of organic fibers and mineral particles. C: Diffraction pattern obtained from the endocuticle demonstrating the amorphous nature of the mineral within it. 